\title{
Design of Wideband MIMO Car-to-Car Channel Models Based on the Geometrical Street Scattering Model
}

\author{
Nurilla Avazov and Matthias Pätzold \\ Faculty of Engineering and Science, University of Agder, P.O. Box 509, 4898 Grimstad, Norway \\ Correspondence should be addressed to Nurilla Avazov, nurilla.k.avazov@uia.no \\ Received 2 May 2012; Revised 28 August 2012; Accepted 5 September 2012 \\ Academic Editor: Neji Youssef
}

Copyright ( $) 2012$ N. Avazov and M. Pätzold. This is an open access article distributed under the Creative Commons Attribution License, which permits unrestricted use, distribution, and reproduction in any medium, provided the original work is properly cited.

\begin{abstract}
We propose a wideband multiple-input multiple-output (MIMO) car-to-car (C2C) channel model based on the geometrical street scattering model. Starting from the geometrical model, a MIMO reference channel model is derived under the assumption of single-bounce scattering in line-of-sight (LOS) and non-LOS (NLOS) propagation environments. The proposed channel model assumes an infinite number of scatterers, which are uniformly distributed in two rectangular areas located on both sides of the street. Analytical solutions are presented for the space-time-frequency cross-correlation function (STF-CCF), the two-dimensional (2D) space CCF, the time-frequency CCF (TF-CCF), the temporal autocorrelation function (ACF), and the frequency correlation function (FCF). An efficient sum-of-cisoids (SOCs) channel simulator is derived from the reference model. It is shown that the temporal ACF and the FCF of the SOC channel simulator fit very well to the corresponding correlation functions of the reference model. To validate the proposed channel model, the mean Doppler shift and the Doppler spread of the reference model have been matched to real-world measurement data. The comparison results demonstrate an excellent agreement between theory and measurements, which confirms the validity of the derived reference model. The proposed geometry-based channel simulator allows us to study the effect of nearby street scatterers on the performance of $\mathrm{C} 2 \mathrm{C}$ communication systems.
\end{abstract}

\section{Introduction}

$\mathrm{C} 2 \mathrm{C}$ communications is an emerging technology, which receives considerable attention due to new traffic telematic applications that improve the efficiency of traffic flow and reduce the number of road accidents [1]. The development of C2C communication technologies is supported in Europe by respected organizations, such as the European Road Transport Telematics Implementation Coordinating Organization (ERTICO) [2] and the C2C Communication Consortium (C2C-CC) [3]. In this context, a large number of research projects focussing on $\mathrm{C} 2 \mathrm{C}$ communications are currently being carried out throughout the world.

In $\mathrm{C} 2 \mathrm{C}$ communication systems, the underlying radio channel differs from traditional fixed-to-mobile and mobileto-fixed channels in the way that both the transmitter and the receiver are in motion. In this connection, robust and reliable traffic telematic systems have to be developed and tested, which calls for new channel models for $\mathrm{C} 2 \mathrm{C}$ communication systems. Furthermore, MIMO communication systems can also be of great interest for $\mathrm{C} 2 \mathrm{C}$ communications due to their higher throughput [4]. In this regard, several MIMO mobileto-mobile (M2M) channel models have been developed and analyzed under different scattering conditions induced by, for example, the two-ring model [5], the elliptical model [6], the T-junction model [7], and the geometrical street model $[8,9]$. A 2D reference model for narrowband singleinput single-output (SISO) M2M Rayleigh fading channels has been proposed by Akki and Haber in [10, 11]. Simulation models for SISO M2M channels have been reported in [12, $13]$. In $[5,14,15]$, the $2 \mathrm{D}$ reference and simulation models have been presented for narrowband MIMO M2M channels. The proposed model in [15] combines the two-ring model and the elliptical model, where a combination of single- and double-bounce scattering in LOS propagation environments is assumed.

All aforementioned channel models are narrowband M2M channel models. In contrast with narrowband channels, a channel is called a wideband channel or frequencyselective channel if the signal bandwidth significantly exceeds 
the coherence bandwidth of the channel. Owing to increasing demands for high data rate wideband communication systems employing MIMO technologies, such as MIMO orthogonal frequency division multiplexing (OFDM) systems, it is of crucial importance to have accurate and realistic wideband MIMO M2M channel models. According to IEEE 802.11p [16], the dedicated frequency bands for short-range communications [17] will be between $5770 \mathrm{MHz}$ and $5925 \mathrm{MHz}$ depending on the region. The range 5795$5815 \mathrm{MHz}$ will be devoted to Europe, while $5850-5925 \mathrm{MHz}$ and $5770-5850 \mathrm{MHz}$ will be assigned to North America and Japan, respectively. Consequently, a large number of $\mathrm{C} 2 \mathrm{C}$ channel measurements have been carried out at different frequency bands, for example, at $2.4 \mathrm{GHz}$ [18], $3.5 \mathrm{GHz}$ [19], $5 \mathrm{GHz}[20,21], 5.2 \mathrm{GHz}[22]$, and $5.9 \mathrm{GHz}$ [23]. Realworld measurement campaigns for wideband $\mathrm{C} 2 \mathrm{C}$ channels can be found in [24-27]. In the literature, there exist several papers [28-30] with the focus on the modeling of wideband MIMO M2M channels. A reference model derived from the geometrical T-junction scattering model has been proposed in [7] for wideband MIMO vehicle-tovehicle (V2V) fading channels. In [29], a three-dimensional (3D) model for a wideband MIMO M2M channel has been studied. Its corresponding first- and second-order statistics have been investigated and validated on the basis of realworld measurement data. In the same paper, it has been shown that $3 \mathrm{D}$ scattering scenarios are more realistic than $2 \mathrm{D}$ scattering scenarios. However, 2D scattering models are more complexity efficient, and they provide a good approximation to $3 \mathrm{D}$ scattering models [31]. For those reasons, we propose in our paper a 2D street scattering model.

In the literature, numerous fundamental channel models with different scatterer distributions, such as the uniform, Gaussian, Laplacian, and von Mises distribution, have been proposed to characterize the angle-of-departure (AOD) and the angle-of-arrival (AOA) statistics. In [32], the author studied the effect of Gaussian distributed scatterers on the channel characteristics in a circular scattering region around a mobile station. The spatial and temporal properties of the first arrival path in multipath environments have also been analyzed in [32]. The authors of [9] assume rectangular scattering areas on both sides of the street, in which an infinite number of scatterers are uniformly distributed. It has been observed that the shape of the Doppler power spectral density (PSD) resembles a Gaussian function if the width of the scattering area is very large.

In contrast to our previous work in [9], where the focus was on the derivation of a reference channel model for narrowband SISO C2C channels, we design in this paper a wideband MIMO C2C channel model by starting from the same geometrical street scattering model. We focus on the statistical characterization of a wideband reference channel model assuming that an infinite number of scatterers are uniformly distributed within two rectangular areas. The radio propagation phenomena in street environments are modelled by a wide-sense stationary uncorrelated scattering process, where in addition a LOS component is taken into account. The reference model has been derived from the geometrical street scattering model assuming that the AOD and the AOA are dependent due to single-bounce scattering. To account for the nature of $\mathrm{C} 2 \mathrm{C}$ channels, we take the mobility of both the transmitter and the receiver for granted.

In our model, we consider a $2 \mathrm{D}$ street scattering environment to reduce the computational cost by still guaranteeing a good match between the reference model and measured channels. A typical propagation scenario for the proposed model is illustrated in Figure 1, where the buildings and the trees are considered as scattering objects. Such a typical dense urban environment scenario allows us to assume that the local scatterers are uniformly distributed in a specific area. An analytical expression will be derived for the STF-CCF from which the 2D space CCF, the TF-CCF, the temporal ACF, and the FCF can be obtained directly. To validate the proposed reference model, the mean Doppler shift and the Doppler spread of the reference model have been matched to the corresponding quantities of the measured channel described in [25] for different propagation environments, such as urban, rural, and highway areas. Furthermore, we have derived an SOC channel simulator from the reference model. It is shown that the designed channel simulator matches the underlying reference model with respect to the temporal ACF and the FCF.

The rest of this paper is organized as follows. Section 2 describes the geometrical street scattering model. In Section 3, the reference channel model is derived from the geometrical street model. Section 4 analyzes the correlation properties of the reference model, such as the STF-CCF, the $2 \mathrm{D}$ space CCF, the TF-CCF, the temporal ACF, and the FCF. The computation of the measurement-based model parameters and the characteristic quantities describing the Doppler effect are discussed in Section 5. Section 6 describes briefly the simulation model derived from the reference model. The illustration of some numerical results found for the correlation functions of the reference model and the corresponding simulation model is the topic of Section 7. Finally, Section 8 draws the conclusion of the paper.

\section{The Geometrical Street Scattering Model}

This section briefly describes the geometrical street scattering model for wideband MIMO C2C channels. The proposed geometrical model describes the scattering environment in an urban area, where the scatterers are located in two rectangular areas on both sides of the street as illustrated in Figure 2. We consider rectangular grids formed by rows and columns, where the length and the width of the rectangular grids are denoted by $L_{A}=A_{1}+A_{2}$ and $B_{i}(i=1,2)$, respectively. The scatterer located in the $m$ th column of the $n$th row is denoted by $S^{(m n)}(m=1,2, \ldots, M, n=$ $1,2, \ldots, N)$. It is assumed that the local scatterers $S^{(m n)}$ are uniformly distributed in the rectangles. The symbols $M S_{T}$ and $M S_{R}$ in Figure 2 stand for the mobile transmitter and the mobile receiver, respectively. The symbol $D$ represents the scalar projection of the distance between the transmitter and the receiver onto the $x$-axis. The transmitter (receiver) is located at a distance $y_{T_{1}}\left(y_{R_{1}}\right)$ from the left-hand side of the street and at a distance $y_{T_{2}}\left(y_{R_{2}}\right)$ from the right-hand side of the street. Both the transmitter and the receiver are in motion 


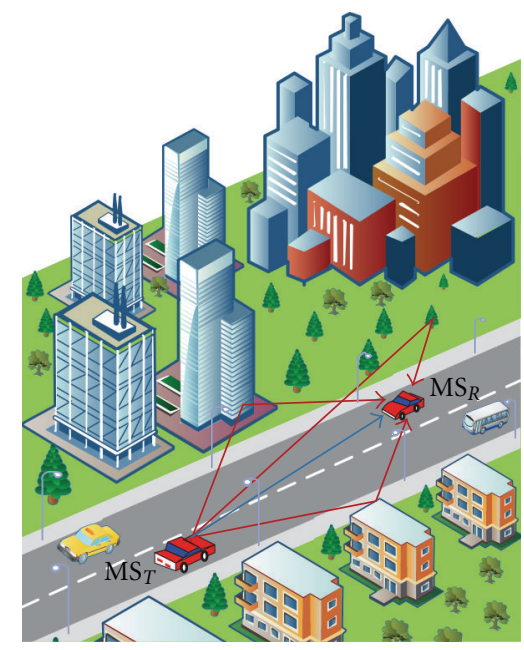

FIGURE 1: A typical propagation scenario along a straight street in urban areas.

and equipped with $M_{T}$ transmitter antenna elements and $M_{R}$ receiver antenna elements, respectively. The antenna element spacings at the transmitter and the receiver are denoted by $\delta_{T}$ and $\delta_{R}$, respectively. The symbols $\alpha_{T}^{(m n)}$ and $\alpha_{R}^{(m n)}$ denote the AOD and the AOA, respectively. The angle $\gamma_{T}\left(\gamma_{R}\right)$ describes the tilt angle of the transmitter (receiver) antenna array. Moreover, it is assumed that the transmitter (receiver) moves with speed $v_{T}\left(v_{R}\right)$ in the direction determined by the angle of motion $\varphi_{v}^{T}\left(\varphi_{v}^{R}\right)$.

\section{The Reference Model}

3.1. Derivation of the Reference Model. In this section, we derive the reference model for the MIMO C2C channel under the assumption of LOS and NLOS propagation conditions. From Figure 2, we realize that the $(m n)$ th homogeneous plane wave emitted from the $l$ th antenna element $A_{T}^{(l)}(l=$ $\left.1,2, \ldots, M_{T}\right)$ of the transmitter travels over the local scatterer $S^{(m n)}$ before impinging on the $k$ th antenna element $A_{R}^{(k)}(k=$ $\left.1,2, \ldots, M_{R}\right)$ of the receiver. The reference model is based on the assumption that the number of local scatterers within both rectangular areas is infinite, that is, $M, N \rightarrow \infty$. The temporal, spatial, and frequency characteristics of the reference model are determined by the $M_{R} \times M_{T}$ channel matrix $\mathbf{H}\left(f^{\prime}, t\right)=\left[H_{k l}\left(f^{\prime}, t\right)\right]_{M_{R} \times M_{T}}$, where $H_{k l}\left(f^{\prime}, t\right)$ denotes the time-variant transfer function (TVTF) of the channel for the link between the $l$ th transmitter antenna element $A_{T}^{(l)}$ and the $k$ th receiver antenna element $A_{R}^{(k)}$. The TVTF $H_{k l}\left(f^{\prime}, t\right)$ can be expressed as a superposition of the diffuse component and the LOS component as follows:

$$
H_{k l}\left(f^{\prime}, t\right)=H_{k l}^{\mathrm{DIF}}\left(f^{\prime}, t\right)+H_{k l}^{\mathrm{LOS}}\left(f^{\prime}, t\right),
$$

where $H_{k l}^{\mathrm{DIF}}\left(f^{\prime}, t\right)$ and $H_{k l}^{\mathrm{LOS}}\left(f^{\prime}, t\right)$ represent the diffuse and the LOS components of the channel, respectively.

Note that the single-bounce scattering components bear more energy than the double-bounce scattering components. Hence, in our analysis, we model the diffuse component $H_{k l}^{\mathrm{DIF}}\left(f^{\prime}, t\right)$ by only taking into account the single-bounce scattering effects, which is in accordance with the assumptions made in $[28,33]$. From the geometrical street scattering model shown in Figure 2, we can derive the TVTF of the diffuse component, which results in the following expression:

$$
\begin{aligned}
H_{k l}^{\mathrm{DIF}}\left(f^{\prime}, t\right)= & \lim _{M, N \rightarrow \infty} \frac{1}{\sqrt{\left(c_{R}+1\right) M N}} \sum_{m, n=1}^{M, N} a_{l, m n} b_{k, m n} c_{m n} \\
& \cdot e^{j\left[\theta_{m n}+2 \pi\left(f_{T}^{(m n)}+f_{R}^{(m n)}\right) t-2 \pi f^{\prime} \tau^{\prime(m l)}\right]},
\end{aligned}
$$

where

$$
\begin{gathered}
a_{l, m n}=e^{j \pi\left(\delta_{T} / \lambda\right)\left(M_{T}-2 l+1\right) \cos \left(\alpha_{T}^{(m n)}-\gamma_{T}\right)}, \\
b_{k, m n}=e^{j \pi\left(\delta_{R} / \lambda\right)\left(M_{R}-2 k+1\right) \cos \left(\alpha_{R}^{(m n)}-\gamma_{R}\right)}, \\
c_{m n}=e^{-j(2 \pi / \lambda)\left(y_{T_{1}} / \sin \left(\alpha_{T}^{(m n)}\right)+y_{R_{1}} / \sin \left(\alpha_{R}^{(m n)}\right)\right)}, \\
f_{T}^{(m n)}=f_{T_{\max }} \cos \left(\alpha_{T}^{(m n)}-\varphi_{v}^{T}\right), \\
f_{R}^{(m n)}=f_{R_{\max }} \cos \left(\alpha_{R}^{(m n)}-\varphi_{v}^{R}\right), \\
\tau_{k l}^{\prime(m n)}=\frac{1}{c_{0}}\left[D_{T}^{(l, m n)}+D_{R}^{(m n, k)}\right] .
\end{gathered}
$$

In (6) and (7), the symbols $f_{T_{\max }}=v_{T} / \lambda$ and $f_{R_{\max }}=$ $v_{R} / \lambda$ denote the maximum Doppler frequencies associated with the movement of the transmitter and the receiver, respectively, and $\lambda$ is the wavelength. The symbol $c_{R}$ in (2) represents the Rice factor, which is defined as the ratio of the power of the LOS component to the power of the diffuse component, that is, $c_{R}=$ $E\left\{\left|H_{k l}^{\mathrm{LOS}}\left(f^{\prime}, t\right)\right|^{2}\right\} / E\left\{\left|H_{k l}^{\mathrm{DIF}}\left(f^{\prime}, t\right)\right|^{2}\right\}$. The phases $\theta_{m n}$ in (2) denote the phase shift introduced by the scatterer $S^{(m n)}$. It is assumed that the phases $\theta_{m n}$ are independent, identically distributed (i.i.d.) random variables, which are uniformly distributed over the interval $[0,2 \pi)$. The symbols $\tau_{k l}^{\prime(m n)}$ and $c_{0}$ represent the propagation delays of the diffuse component and the speed of light, respectively. In (8), the quantity $D_{T}^{(l, m n)}$ 


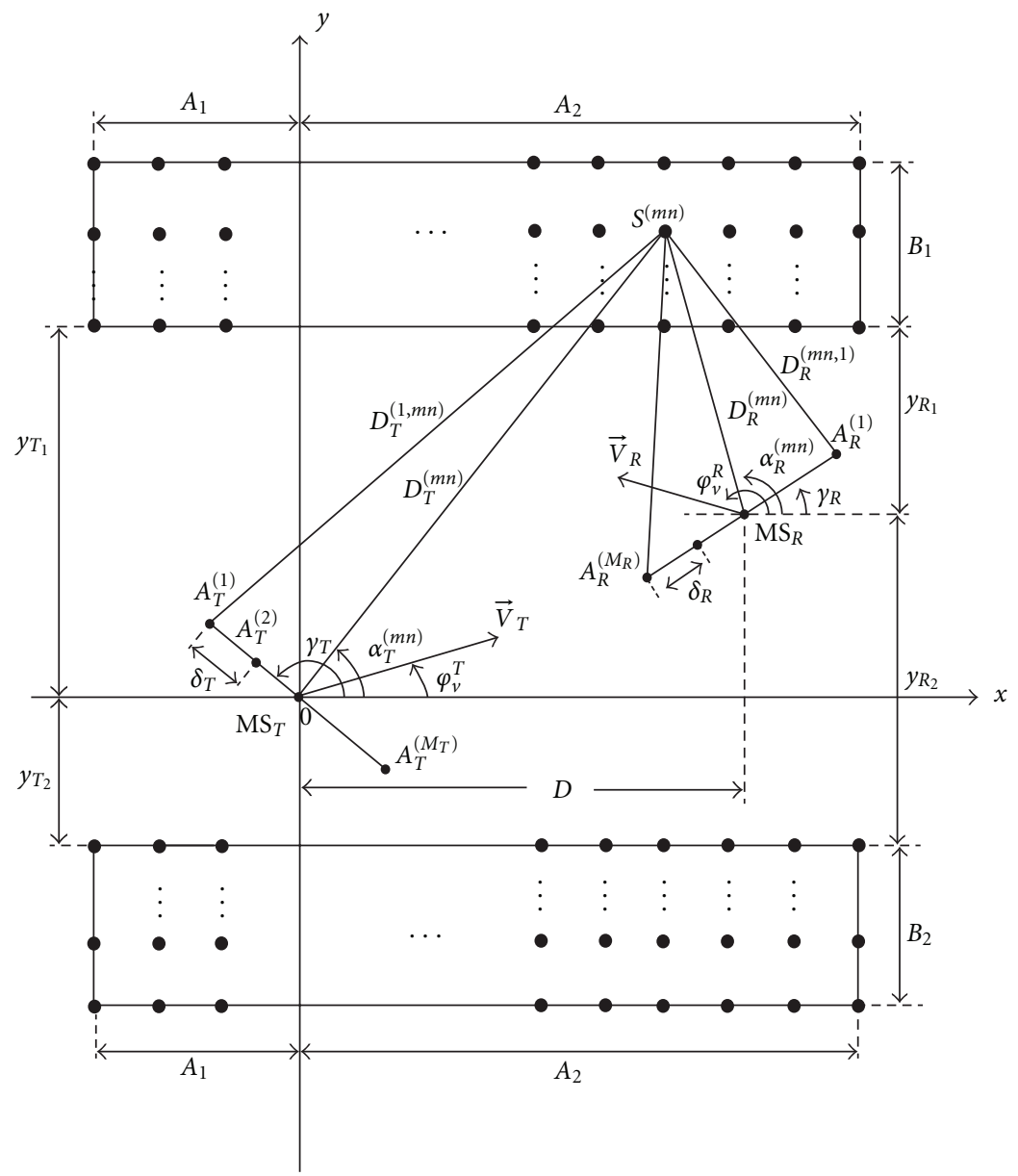

FIGURE 2: The geometrical street scattering model with local scatterers uniformly distributed in two rectangular areas on both sides of the street.

stands for the distance from the $l$ th transmitter antenna element $A_{T}^{(l)}$ to the scatterer $S^{(m n)}$, whereas $D_{R}^{(m n, k)}$ is the distance between the scatterer $S^{(m n)}$ and the $k$ th receiver antenna element $A_{R}^{(k)}$. It is assumed that $\left(M_{T}-1\right) \delta_{T} \ll$ $\min \left\{y_{T 1}, y_{T 2}\right\}$ and $\left(M_{R}-1\right) \delta_{R} \ll \min \left\{y_{R 1}, y_{R 2}\right\}$. These assumptions, together with the approximation $\sqrt{1+x} \approx$ $1+x / 2(x \ll 1)$, allow us to approximate the two distances $D_{T}^{(l, m n)}$ and $D_{R}^{(m n, k)}$ as follows:

$$
\begin{aligned}
D_{T}^{(l, m n)} \approx & D_{T}^{(m n)} \\
& -\left(M_{T}-2 l+1\right)\left(\frac{\delta_{T}}{2}\right) \cos \left(\alpha_{T}^{(m n)}-\gamma_{T}\right), \\
D_{R}^{(m n, k)} \approx & D_{R}^{(m n)}-\left(M_{R}-2 k+1\right)\left(\frac{\delta_{R}}{2}\right) \cos \left(\alpha_{R}^{(m n)}-\gamma_{R}\right),
\end{aligned}
$$

where $D_{T}^{(m n)}$ and $D_{R}^{(m n)}$ are given by $D_{T}^{(m n)}=y_{T_{1}} / \sin \left(\alpha_{T}^{(m n)}\right)$ and $D_{R}^{(m n)}=y_{R_{1}} / \sin \left(\alpha_{R}^{(m n)}\right)$, respectively.

It is noteworthy that one can also find articles [11, 34], in which only double-bounce scattering is assumed for M2M communications. However, by following a similar approach as in [15], one can easily extend our analysis on the basis of single-bounce scattering to the case of double-bounce scattering, and thus also to a combination of single- and double-bounce scattering.

The TVTF of the LOS component is given by

$$
H_{k l}^{\mathrm{LOS}}\left(f^{\prime}, t\right)=\sqrt{\frac{c_{R}}{\left(c_{R}+1\right)}} e^{j\left[2 \pi\left(f_{T}^{(0)}+f_{R}^{(0)}\right) t-(2 \pi / \lambda) D_{k l}-2 \pi f^{\prime} \tau_{k l}^{\prime(0)}\right]},
$$

where

$$
\begin{gathered}
f_{T}^{(0)}=f_{T_{\max }} \cos \left(\alpha_{T}^{(0)}-\varphi_{v}^{T}\right), \\
f_{R}^{(0)}=f_{R_{\max }} \cos \left(\alpha_{R}^{(0)}-\varphi_{v}^{R}\right), \\
D_{k l}=D_{0}-\left(M_{T}-2 l+1\right) \frac{\delta_{R}}{2} \cos \left(\gamma_{T}\right) \\
+\left(M_{R}-2 k+1\right) \frac{\delta_{R}}{2} \cos \left(\gamma_{R}\right), \\
D_{0}=\sqrt{D^{2}+\left(y_{T_{1}}-y_{R_{1}}\right)^{2}} .
\end{gathered}
$$

In (11), $f_{T}^{(0)}$ and $f_{R}^{(0)}$ denote the Doppler shifts of the LOS component caused by the movement of the transmitter and the receiver, respectively. The symbols $\alpha_{T}^{(0)}$ and $\alpha_{R}^{(0)}$ 
in (12) and (13) represent the AOD and the AOA of the LOS component, respectively. Finally, $\tau_{k l}^{\prime(0)}$ denotes the propagation delay of the LOS component. The delay of the LOS component is defined by $\tau_{k l}^{\prime(0)}=D_{k l} / c_{0}$ with $D_{k l}$ being the length of the direct path from the $l$ th transmitter antenna element $A_{T}^{(l)}$ to the $k$ th receiver antenna element $A_{R}^{(k)}$. The symbol $D_{0}$ in (14) denotes the Euclidean distance between the transmitter and the receiver. According to [35], the LOS component $H_{k l}^{\mathrm{LOS}}\left(f^{\prime}, t\right)$ is assumed to be a deterministic process, while the diffuse component $H_{k l}^{\mathrm{DIF}}\left(f^{\prime}, t\right)$ is a stochastic process.

3.2. Derivation of the AOD and the AOA. The position of all local scatterers $S^{(m n)}$ is described by the Cartesian coordinates $\left(x_{m}, y_{n}\right)$. In the reference model, the coordinates $x_{m}$ and $y_{n}$ are independent random variables, which are determined by the distribution of the local scatterers. With reference to Figure 2, we take into account that due to single-bounce scattering, the $\operatorname{AOD} \alpha_{T}^{(m n)}$ and the $\operatorname{AOA} \alpha_{R}^{(m n)}$ are dependent. By using the trigonometric identities, we can express the AOD $\alpha_{T}^{(m n)}$ and the AOA $\alpha_{R}^{(m n)}$ in terms of the coordinates $\left(x_{m}, y_{n}\right)$ of the local scatterers $S^{(m n)}$ as follows:

$$
\begin{aligned}
& \alpha_{T}^{(m n)}\left(x_{m}, y_{n}\right) \\
& \quad= \begin{cases}g\left(x_{m}, y_{n}\right), & \text { if } y_{n} \in J_{i}, x_{m} \in\left[0, A_{2}\right] \\
(-1)^{i+1} \pi+g\left(x_{m}, y_{n}\right), & \text { if } y_{n} \in J_{i}, x_{m} \in\left[-A_{1}, 0\right]\end{cases}
\end{aligned}
$$

$$
\begin{aligned}
& \alpha_{R}^{(m n)}\left(x_{m}, y_{n}\right) \\
& \quad= \begin{cases}f\left(x_{m}, y_{n}\right), & \text { if } y_{n} \in J_{i}, x_{m} \in\left[D, A_{2}\right] \\
(-1)^{i+1} \pi+f\left(x_{m}, y_{n}\right), & \text { if } y_{n} \in J_{i}, x_{m} \in\left[-A_{1}, D\right]\end{cases}
\end{aligned}
$$

for $i=1,2$, where $J_{1}=\left[y_{T_{1}}, y_{T_{1}}+B_{1}\right], J_{2}=\left[-y_{T_{2}}-B_{2},-y_{T_{2}}\right]$, and

$$
\begin{gathered}
g\left(x_{m}, y_{n}\right)=\arctan \frac{y_{n}}{x_{m}} \\
f\left(x_{m}, y_{n}\right)=\arctan \frac{y_{n}-y_{T_{1}}+y_{R_{1}}}{x_{m}-D} .
\end{gathered}
$$

\section{Correlation Properties of the Reference Model}

In this section, we derive a general analytical solution for the STF-CCF, from which other correlation functions, such as the $2 \mathrm{D}$ space CCF, the TF-CCF, the temporal ACF, and the FCF can easily be derived.

4.1. Derivation of the STF-CCF. According to [10], the STFCCF of the links $A_{T}^{(l)}-A_{R}^{(k)}$ and $A_{T}^{\left(l^{\prime}\right)}-A_{R}^{\left(k^{\prime}\right)}$ is defined as the correlation between the channel transfer functions $H_{k l}\left(f^{\prime}, t\right)$ and $H_{k^{\prime} l^{\prime}}\left(f^{\prime}, t\right)$, that is,

$$
\begin{aligned}
\rho_{k l, k^{\prime} l^{\prime}}\left(\delta_{T}, \delta_{R}, v^{\prime}, \tau\right)= & E\left\{H_{k l}^{*}\left(f^{\prime}, t\right) H_{k^{\prime} l^{\prime}}\left(f^{\prime}+v^{\prime}, t+\tau\right)\right\} \\
= & \rho_{k l, k^{\prime} l^{\prime}}^{\operatorname{DIF}}\left(\delta_{T}, \delta_{R}, v^{\prime}, \tau\right) \\
& +\rho_{k l, k^{\prime} l^{\prime}}^{\mathrm{LOS}}\left(\delta_{T}, \delta_{R}, v^{\prime}, \tau\right),
\end{aligned}
$$

where $(*)$ denotes the complex conjugate operator and $E\{\cdot\}$ stands for the expectation operator that applies to all random variables: the phases $\left\{\theta_{m n}\right\}$ and the coordinates $\left(x_{m}, y_{n}\right)$ of the scatterers $S^{(m n)}$. The first term $\rho_{k l, k^{\prime} l^{\prime}}^{\mathrm{DIF}}\left(\delta_{T}, \delta_{R}, \tau, v^{\prime}\right)$ represents the STF-CCF of the diffuse component. This correlation function can be expressed, after substituting (2) in (19), by

$$
\begin{aligned}
\rho_{k l, k^{\prime} l^{\prime}}^{\mathrm{DIF}} & \left.\delta_{T}, \delta_{R}, v^{\prime}, \tau\right) \\
= & \lim _{M, N \rightarrow \infty} \frac{1}{\left(c_{R}+1\right) M N} \\
& \times \sum_{m, n=1}^{M, N} E\left\{c_{l l^{\prime}}^{(m n)} d_{k k^{\prime}}^{(m n)} e^{j 2 \pi\left[\left(f_{T}^{(m n)}+f_{R}^{(m n)}\right) \tau-\nu^{\prime} \tau_{k l}^{(m n)}\right]}\right\},
\end{aligned}
$$

where

$$
\begin{aligned}
& c_{l l^{\prime}}^{(m n)}=e^{j 2 \pi\left(\delta_{T} / \lambda\right)\left(l-l^{\prime}\right) \cos \left(\alpha_{T}^{(m n)}-\gamma_{T}\right)}, \\
& d_{k k^{\prime}}^{(m n)}=e^{j 2 \pi\left(\delta_{R} / \lambda\right)\left(k-k^{\prime}\right) \cos \left(\alpha_{R}^{(m n)}-\gamma_{R}\right)} .
\end{aligned}
$$

The quantities $f_{T}^{(m n)}, f_{R}^{(m n)}$, and $\tau_{k l}^{\prime(m n)}$ are given by (6), (7), and (8), respectively. We recall that the AOD $\alpha_{T}^{(m n)}$ and the AOA $\alpha_{R}^{(m n)}$ can be expressed in terms of the random variables $x_{m}$ and $y_{n}$ according to (16) and (17), respectively.

In Section 2, it has been mentioned that all scatterers are uniformly distributed in the two rectangular areas on both sides of the street, as illustrated in Figure 2. Hence, the random variables $x_{m}$ and $y_{n}$ are also uniformly distributed over the rectangular areas. If the number of scatterers tends to infinity, that is, $M, N \rightarrow \infty$, then the discrete random variables $x_{m}$ and $y_{n}$ become continuous random variables denoted by $x$ and $y$, respectively. Thus, the probability density functions (PDFs) $p_{x}(x)$ and $p_{y}(y)$ of $x$ and $y$, respectively, are given by

$$
\begin{gathered}
p_{x}(x)=\frac{1}{L_{A}}, \quad \text { if } x \in\left[-A_{1}, A_{2}\right], \\
p_{y}(y)= \begin{cases}\frac{1}{2 B_{1}}, & \text { if } y \in\left[y_{T_{1}}, B_{1}+y_{T_{1}}\right] \\
\frac{1}{2 B_{2}}, & \text { if } y \in\left[-B_{2}-y_{T_{2}},-y_{T_{2}}\right],\end{cases}
\end{gathered}
$$

where $L_{A}=A_{1}+A_{2}$. Assuming that the random variables $x$ and $y$ are independent, the joint $\operatorname{PDF} p_{x y}(x, y)$ of the 
random variables $x$ and $y$ can be expressed as a product of the marginal PDFs $p_{x}(x)$ and $p_{y}(y)$, that is,

$$
\begin{aligned}
& p_{x y}(x, y) \\
& \quad=p_{x}(x) \cdot p_{y}(y) \\
& \quad= \begin{cases}\frac{1}{2 L_{A} B_{1}}, & \text { if } x \in\left[-A_{1}, A_{2}\right], y \in\left[y_{T_{1}}, B_{1}+y_{T_{1}}\right] \\
\frac{1}{2 L_{A} B_{2}}, & \text { if } x \in\left[-A_{1}, A_{2}\right], y \in\left[-B_{2}-y_{T_{2}},-y_{T_{2}}\right] .\end{cases}
\end{aligned}
$$

The infinitesimal power of the diffuse component corresponding to the differential axes $d x$ and $d y$ is proportional to $p_{x y}(x, y) d x d y$. As $M, N \rightarrow \infty$, this infinitesimal contribution must be equal to $1 / M N=p_{x y}(x, y) d x d y$. Consequently, it follows from (20) that the STF-CCF of the diffuse component can be expressed as

$$
\begin{aligned}
\rho_{k l, k^{\prime} l^{\prime}}^{\mathrm{DIF}} & \left(\delta_{T}, \delta_{R}, v^{\prime}, \tau\right) \\
= & \frac{1}{2 L_{A} B_{1}\left(c_{R}+1\right)} \int_{y_{T_{1}}}^{y_{T_{1}}+B_{1}} \int_{-A_{1}}^{A_{2}} c_{l l^{\prime}}^{\mathrm{DIF}}\left(\delta_{T}, x, y\right) d_{k k^{\prime}}^{\mathrm{DIF}}\left(\delta_{R}, x, y\right) \\
& \times e^{\mathrm{j} 2 \pi\left[\left(f_{T}(x, y)+f_{R}(x, y)\right) \tau-\nu^{\prime} \tau_{k l}^{\prime}(x, y)\right]} d x d y \\
& +\frac{1}{2 L_{A} B_{2}\left(c_{R}+1\right)} \\
& \times \int_{-B_{2}-y_{T_{2}}}^{-y_{T_{2}}} \int_{-A_{1}}^{A_{2}} c_{l l^{\prime}}^{\mathrm{DIF}}\left(\delta_{T}, x, y\right) d_{k k^{\prime}}^{\mathrm{DIF}}\left(\delta_{R}, x, y\right) \\
& \times e^{j 2 \pi\left[\left(f_{T}(x, y)+f_{R}(x, y)\right) \tau-\nu^{\prime} \tau_{k l}^{\prime}(x, y)\right]} d x d y,
\end{aligned}
$$

where

$$
\begin{gathered}
c_{l l^{\prime}}^{\mathrm{DIF}}\left(\delta_{T}, x, y\right)=e^{j 2 \pi\left(\delta_{T} / \lambda\right)\left(l-l^{\prime}\right) \cos \left(\alpha_{T}(x, y)-\gamma_{T}\right)}, \\
d_{k k^{\prime}}^{\mathrm{DIF}}\left(\delta_{R}, x, y\right)=e^{j 2 \pi\left(\delta_{R} / \lambda\right)\left(k-k^{\prime}\right) \cos \left(\alpha_{R}(x, y)-\gamma_{R}\right)}, \\
f_{T}(x, y)=f_{T_{\max }} \cos \left(\alpha_{T}(x, y)-\varphi_{v}^{T}\right), \\
f_{R}(x, y)=f_{R_{\max }} \cos \left(\alpha_{R}(x, y)-\varphi_{v}^{R}\right), \\
\tau_{k l}^{\prime}(x, y)=\frac{1}{c_{0}}\left[D_{T}^{(l)}(x, y)+D_{R}^{(k)}(x, y)\right] .
\end{gathered}
$$

Using the functions in (9) and (10), the distances $D_{T}^{(l)}(x, y)$ and $D_{R}^{(k)}(x, y)$ can be expressed as

$$
\begin{aligned}
D_{T}^{(l)}(x, y) \approx & \frac{y_{T_{1}}}{\sin \left(\alpha_{T}(x, y)\right)} \\
& -\left(M_{T}-2 l+1\right)\left(\frac{\delta_{T}}{2}\right) \cos \left(\alpha_{T}(x, y)-\gamma_{T}\right) \\
D_{R}^{(k)}(x, y) \approx & \frac{y_{R_{1}}}{\sin \left(\alpha_{R}(x, y)\right)} \\
& -\left(M_{R}-2 k+1\right)\left(\frac{\delta_{R}}{2}\right) \cos \left(\alpha_{R}(x, y)-\gamma_{R}\right)
\end{aligned}
$$

In (19), the quantity $\rho_{k l, k^{\prime} l^{\prime}}^{\mathrm{LOS}}\left(\delta_{T}, \delta_{R}, \tau, \nu^{\prime}\right)$, which represents the STF-CCF of the LOS component, can be written as

$$
\begin{aligned}
\rho_{k l, k^{\prime} l^{\prime}}^{\mathrm{LOS}}\left(\delta_{T}, \delta_{R}, v^{\prime}, \tau\right)= & \frac{c_{R}}{\left(c_{R}+1\right)} c_{l l^{\prime}}^{(0)}\left(\delta_{T}\right) \\
& \left.\times d_{k k^{\prime}}^{(0)}\left(\delta_{R}\right) e^{j 2 \pi\left[\left(f_{T}^{(0)}+f_{R}^{(0)}\right) \tau-v^{\prime} \tau_{k l}^{\prime}(0)\right.}\right]
\end{aligned}
$$

where

$$
\begin{gathered}
c_{l l^{\prime}}^{(0)}\left(\delta_{T}\right)=e^{j 2 \pi\left(\delta_{T} / \lambda\right)\left(l-l^{\prime}\right) \cos \left(\gamma_{T}\right)}, \\
d_{k k^{\prime}}^{(0)}\left(\delta_{R}\right)=e^{-j 2 \pi\left(\delta_{R} / \lambda\right)\left(k-k^{\prime}\right) \cos \left(\gamma_{R}\right)} .
\end{gathered}
$$

The Doppler shifts $f_{T}^{(0)}$ and $f_{R}^{(0)}$ are given by (12) and (13), respectively.

4.2. Derivation of the $2 D$ Space CCF. The $2 \mathrm{D}$ space CCF $\rho_{k l, k^{\prime} l^{\prime}}\left(\delta_{T}, \delta_{R}\right)$ is defined as $\rho_{k l, k^{\prime} l^{\prime}}\left(\delta_{T}, \delta_{R}\right)=$ $E\left\{H_{k l}^{*}\left(f^{\prime}, t\right) H_{k^{\prime} l^{\prime}}\left(f^{\prime}, t\right)\right\}$, which is equal to the STF-CCF $\rho_{k l, k^{\prime} l^{\prime}}\left(\delta_{T}, \delta_{R}, \nu^{\prime}, \tau\right)$ in (19) by setting $\nu^{\prime}$ and $\tau$ to zero, that is,

$$
\begin{aligned}
\rho_{k l, k^{\prime} l^{\prime}}\left(\delta_{T}, \delta_{R}\right)= & \rho_{k l, k^{\prime} l^{\prime}}\left(\delta_{T}, \delta_{R}, 0,0\right) \\
= & \frac{1}{2 L_{A} B_{1}\left(c_{R}+1\right)} \int_{y_{T_{1}}}^{y_{T_{1}}+B_{1}} \\
& \times \int_{-A_{1}}^{A_{2}} c_{l l^{\prime}}^{\mathrm{DIF}}\left(\delta_{T}, x, y\right) d_{k k^{\prime}}^{\mathrm{DIF}}\left(\delta_{R}, x, y\right) d x d y \\
& +\frac{1}{2 L_{A} B_{2}\left(c_{R}+1\right)} \int_{-B_{2}-y_{T_{2}}}^{-y_{T_{2}}} \\
& \times \int_{-A_{1}}^{A_{2}} c_{l l^{\prime}}^{\mathrm{DIF}}\left(\delta_{T}, x, y\right) d_{k k^{\prime}}^{\mathrm{DIF}}\left(\delta_{R}, x, y\right) d x d y \\
& +\frac{c_{R}}{\left(c_{R}+1\right)} c_{l l^{\prime}}^{(0)}\left(\delta_{T}\right) d_{k k^{\prime}}^{(0)}\left(\delta_{R}\right) .
\end{aligned}
$$

4.3. Derivation of the TF-CCF. The TF-CCF of the transmission link from $A_{T}^{(l)}\left(l=1,2, \ldots, M_{T}\right)$ to $A_{R}^{(k)} \quad(k=$ $\left.1,2, \ldots, M_{R}\right)$ is defined by $r_{k l}\left(v^{\prime}, \tau\right):=E\left\{H_{k l}^{*}\left(f^{\prime}, t\right) H_{k l}\left(f^{\prime}+\right.\right.$ $\left.\left.v^{\prime}, t+\tau\right)\right\}$ [36]. The TF-CCF can be obtained directly from the STF-CCF [see (19)] by setting the antenna element spacings $\delta_{T}$ and $\delta_{R}$ to zero, that is,

$$
\begin{aligned}
r_{k l}\left(v^{\prime}, \tau\right)= & \rho_{k l, k^{\prime} l^{\prime}}^{\mathrm{DIF}}\left(0,0, v^{\prime}, \tau\right)+\rho_{k l, k^{\prime} l^{\prime}}^{\mathrm{LOS}}\left(0,0, v^{\prime}, \tau\right) \\
= & \frac{1}{2 L_{A} B_{1}\left(c_{R}+1\right)} \int_{y_{T_{1}}}^{y_{T_{1}}+B_{1}} \\
& \times \int_{-A_{1}}^{A_{2}} e^{j 2 \pi\left[\left(f_{T}(x, y)+f_{R}(x, y)\right) \tau-\nu^{\prime} \tau_{k l}^{\prime}(x, y)\right]} d x d y \\
& +\frac{1}{2 L_{A} B_{2}\left(c_{R}+1\right)} \int_{-B_{2}-y_{T_{2}}}^{-y_{T_{2}}} \\
& \times \int_{-A_{1}}^{A_{2}} e^{j 2 \pi\left[\left(f_{T}(x, y)+f_{R}(x, y)\right) \tau-\nu^{\prime} \tau_{k l}^{\prime}(x, y)\right]} d x d y \\
& +\frac{c_{R}}{\left(c_{R}+1\right)} e^{j 2 \pi\left(f_{T}^{(0)}+f_{R}^{(0)}\right) \tau} e^{-j 2 \pi v^{\prime} \tau_{k l}^{\prime}(0)} .
\end{aligned}
$$


4.4. Derivation of the Temporal ACF and the Doppler PSD. The temporal ACF of the transmission link from $A_{T}^{(l)}(l=$ $\left.1,2, \ldots, M_{T}\right)$ to $A_{R}^{(k)}\left(k=1,2, \ldots, M_{R}\right)$ is defined by $r_{k l}(\tau):=$ $E\left\{H_{k l}^{*}\left(f^{\prime}, t\right) H_{k l}\left(f^{\prime}, t+\tau\right)\right\}$ [36, Page 376]. The temporal ACF can be obtained directly from the TF-CCF (see (31)) by setting at $v^{\prime}$ to zero, that is, $r_{k l}(\tau)=r_{k l}(\tau, 0)$, which gives

$$
\begin{aligned}
r_{k l}(\tau)= & \frac{1}{2 L_{A} B_{1}\left(c_{R}+1\right)} \int_{y_{T_{1}}}^{y_{T_{1}}+B_{1}} \\
& \times \int_{-A_{1}}^{A_{2}} e^{j 2 \pi\left[f_{T}(x, y)+f_{R}(x, y)\right] \tau} d x d y \\
& +\frac{1}{2 L_{A} B_{2}\left(c_{R}+1\right)} \int_{-B_{2}-y_{T_{2}}}^{-y_{T_{2}}} \\
& \times \int_{-A_{1}}^{A_{2}} e^{j 2 \pi\left[f_{T}(x, y)+f_{R}(x, y)\right] \tau} d x d y \\
& +\frac{c_{R}}{\left(c_{R}+1\right)} e^{j 2 \pi\left(f_{T}^{(0)}+f_{R}^{(0)}\right) \tau} .
\end{aligned}
$$

Notice that the expression in (32) reveals that the ACF $r_{k l}(\tau)$ is independent of $k$ and $l$.

Computing the Fourier transform of the temporal ACF $r_{k l}(\tau)$ results in the Doppler PSD $S_{k l}(f)$, that is,

$$
S_{k l}(f)=\int_{-\infty}^{\infty} r_{k l}(\tau) e^{-j 2 \pi f \tau} d \tau .
$$

The two most important statistical quantities characterizing the Doppler PSD $S_{k l}(f)$ are the average Doppler shift $B_{k l}^{(1)}$ and the Doppler spread $B_{k l}^{(2)}$ [35]. The average Doppler shift $B_{k l}^{(1)}$ is defined as the first moment of $S_{k l}(f)$, which can be expressed as follows:

$$
B_{k l}^{(1)}=\frac{\int_{-\infty}^{\infty} f S_{k l}(f) d f}{\int_{-\infty}^{\infty} S_{k l}(f) d f} .
$$

The Doppler spread $B_{k l}^{(2)}$ is defined as the square root of the second central moment of $S_{k l}(f)$, which can be written as

$$
B_{k l}^{(2)}=\sqrt{\frac{\int_{-\infty}^{\infty}\left(f-B_{k l}^{(1)}\right)^{2} S_{k l}(f) d f}{\int_{-\infty}^{\infty} S_{k l}(f) d f}} .
$$

4.5. Derivation of the FCF. The frequency characteristics of the reference model are described by the FCF $r_{k l}\left(\nu^{\prime}\right)$. The FCF $r_{k l}\left(\nu^{\prime}\right)$ of the transmission link from $A_{T}^{(l)}$ to $A_{R}^{(k)}$ is defined by $r_{k l}\left(\nu^{\prime}\right):=E\left\{H_{k l}^{*}\left(f^{\prime}, t\right) H_{k l}\left(f^{\prime}+\nu^{\prime}, t\right)\right\}$ for all $l=1,2, \ldots, M_{T}$ and $k=1,2, \ldots, M_{R}$. This function can be obtained directly from the TF-CCF [see (31)] by setting $\tau$ to zero, that is, $r_{k l}\left(v^{\prime}\right)=r_{k l}\left(0, v^{\prime}\right)$, which results in

$$
\begin{aligned}
r_{k l}\left(\nu^{\prime}\right)= & \frac{1}{2 L_{A} B_{1}\left(c_{R}+1\right)} \int_{y_{T_{1}}}^{y_{T_{1}}+B_{1}} \int_{-A_{1}}^{A_{2}} e^{-j 2 \pi v^{\prime} \tau_{k l}^{\prime}(x, y)} d x d y \\
& +\frac{1}{2 L_{A} B_{2}\left(c_{R}+1\right)} \int_{-B_{2}-y_{T_{2}}}^{-y_{T_{2}}} \int_{-A_{1}}^{A_{2}} e^{-j 2 \pi v^{\prime} \tau_{k l}^{\prime}(x, y)} d x d y \\
& +\frac{c_{R}}{\left(c_{R}+1\right)} e^{-j 2 \pi v^{\prime} \tau_{k l}^{\prime}(0)} .
\end{aligned}
$$

In contrast to the temporal ACF $r_{k l}(\tau)$, the FCF $r_{k l}\left(\nu^{\prime}\right)$ depends on $k$ and $l$.

\section{Measurement-Based Computation of the Model Parameters}

The objective of this section is to determine the set of model parameters $\mathcal{P}=\left\{A_{1}, A_{2}, B_{1}, B_{2}, y_{T_{1}}, y_{T_{2}}, y_{R_{1}}, y_{R_{2}}, D, f_{T_{\text {max }}}\right.$, $\left.f_{R_{\max }}, c_{R}\right\}$ describing the reference model in such a way that the average Doppler shift $B_{k l}^{(1)}$ and the Doppler spread $B_{k l}^{(2)}$ of the reference model match the corresponding quantities $\left(B_{k l}^{\star(1)}\right.$ and $\left.B_{k l}^{\star(2)}\right)$ of the measured channel reported in [25]. To determine the set of model parameters $\mathcal{P}$, we minimize the following error:

$$
E_{\min }=W_{1} E_{B_{k l}^{(1)}}+W_{2} E_{B_{k l}^{(2)}},
$$

where $W_{1}$ and $W_{2}$ denote the weighting factors. The symbols $E_{B_{k l}^{(1)}}$ and $E_{B_{k l}^{(2)}}$ in (37) stand for the absolute errors of the average Doppler shift and Doppler spread, respectively, which are defined as

$$
\begin{aligned}
& E_{B_{k l}^{(1)}}=\arg \min _{\mathcal{P}}\left|B_{k l}^{\star(1)}-B_{k l}^{(1)}\right|, \\
& E_{B_{k l}^{(2)}}=\arg \min _{\mathcal{P}}\left|B_{k l}^{\star(2)}-B_{k l}^{(2)}\right| .
\end{aligned}
$$

In (38) and (39), the notation $\arg \min _{x} f(x)$ stands for the argument of the minimum, which is the set of points of the given argument for which $f(x)$ reaches its minimum value. At the beginning of the optimization procedure, the weighting factors $W_{1}$ and $W_{2}$ are selected arbitrarily, but such that they satisfy the equality $W_{1}+W_{2}=1$. If the error $E_{B_{k l}^{(i)}}(i=1,2)$ in (37) is large, then we reduce the corresponding weighting factor $W_{i}$ and vice versa. We continue the optimization procedure until the result in (37) reaches an error floor, meaning that the average Doppler shift and the Doppler spread of the reference model best match the measured average Doppler shift and the measured Doppler spread, respectively.

For the measured channels in [25], the resulting optimized model parameters and the corresponding average Doppler shift and Doppler spread are listed in Table 1. The results found for the reference model demonstrate an excellent fitting to real-world measured channels for rural, urban, and highway propagation areas, which validates the usefulness of the proposed reference model. It is worth 
TABLE 1: Measurement-based parameters of the geometrical street scattering model and the resulting average Doppler shift and the Doppler spread.

\begin{tabular}{|c|c|c|c|c|c|}
\hline \multirow{2}{*}{ Model parameters } & \multicolumn{5}{|c|}{ Propagation environment } \\
\hline & Urban LOS & Urban NLOS & Rural LOS & Highway LOS & Highway NLOS \\
\hline$A_{1}\left(A_{2}\right)(\mathrm{m})$ & $546.28(1249)$ & $537.03(908.3)$ & $546.52(1236)$ & $547.69(1207)$ & $546.88(1193)$ \\
\hline$B_{1}\left(B_{2}\right)(\mathrm{m})$ & $198.96(198.77)$ & $76.46(1.1113)$ & $20.89(18.25)$ & $199.8(200)$ & $0.01(0.01)$ \\
\hline$f_{T_{\max }}\left(f_{R_{\max }}\right)(\mathrm{Hz})$ & $223.55(219.77)$ & $262.1(209.97)$ & $463.72(491.65)$ & $511.68(442.62)$ & 491.67 (481.97) \\
\hline$y_{T 1}\left(y_{T 2}\right)(\mathrm{m})$ & $10.42(7)$ & $2.12(1.18)$ & $15.28(4.63)$ & $17.62(19.78)$ & $1.3(1.3)$ \\
\hline$y_{R 1}\left(y_{R 2}\right)(\mathrm{m})$ & $19.82(6.6)$ & $20(7.06)$ & $14.57(9.4)$ & $19.63(25)$ & $20(9.4)$ \\
\hline$D(\mathrm{~m})$ & 238.6 & 236.7 & 186.77 & 896.7 & 749.6 \\
\hline$c_{R}$ & 0.485 & 0 & 0.27 & 0.4 & 0 \\
\hline \multicolumn{6}{|l|}{ Measured } \\
\hline \multicolumn{6}{|l|}{$\operatorname{shift} B_{k l}^{\star(1)}(\mathrm{Hz})[25]$} \\
\hline $\begin{array}{l}\text { Theoretical } \\
\text { average Doppler } \\
\text { shift } B_{k l}^{(1)}(\mathrm{Hz})\end{array}$ & -20 & 102.67 & 200.55 & 208.8 & -110 \\
\hline \multicolumn{6}{|l|}{ Measured } \\
\hline \multicolumn{6}{|l|}{$\operatorname{spread} B_{k l}^{\star(2)}(\mathrm{Hz})[25]$} \\
\hline \multicolumn{6}{|l|}{ Theoretical } \\
\hline $\begin{array}{l}\text { Doppler } \\
\text { spread } B_{k l}^{(2)}(\mathrm{Hz})\end{array}$ & 341 & 298 & 782.03 & 760.88 & 941 \\
\hline
\end{tabular}

mentioning that the computed average Doppler shift $B_{k l}^{(1)}=$ $-110 \mathrm{~Hz}$ and the Doppler spread $B_{k l}^{(2)}=941 \mathrm{~Hz}$ do not closely agree with the measured channel $\left(B_{k l}^{\star(1)}=-176 \mathrm{~Hz}\right.$ and $B_{k l}^{\star(2)}=978 \mathrm{~Hz}$ ) in case of the highway NLOS scenario. For this scenario, a close agreement can be found for sufficiently small values of $c_{R} \neq 0$.

\section{The Simulation Model}

The reference model described above is a theoretical model, which is based on the assumption that the number of scatterers $(M, N)$ is infinite. Owing to an infinite realization complexity, the reference model is non-realizable. However, the reference model can serve as a ground for the derivation of stochastic and deterministic simulation models. According to the generalized principle of deterministic channel modeling [35, Sec. 8.1], a stochastic simulation model can be derived from the reference model introduced in (1) by using only a finite number of scatterers. In the literature, several different models exist that allow for a proper simulation of mobile channels. The SOC model is an appropriate simulation model for mobile radio channels under nonisotropic scattering conditions. A detailed description and the design of SOC models can be found in [37, 38], respectively. In [38], several parametrization techniques for SOC models have been discussed and analyzed. Here, we use the $L_{p}$-norm method (LPNM), which is a high-performance parameter computation method for the design of SOC channel simulators.

\section{Numerical Results}

This section illustrates the analytical results given by (30), (31), (32), and (36). The correctness of the analytical results will be verified by simulations. The performance of the channel simulator has been assessed by comparing its temporal ACF and the FCF to the corresponding system functions of the reference model (see (32) and (36)).

As an example for our geometrical street scattering model, we consider rectangular scattering areas on both sides of the street with a length of $L_{A}=A_{1}+A_{2}$, where $A_{1}=$ $50 \mathrm{~m}$ and $A_{2}=450 \mathrm{~m}$, and a width of $B_{1}=B_{2}=100 \mathrm{~m}$. With reference to Figure 2, the position of the transmitter and the receiver are defined by the distances $D=400 \mathrm{~m}$, $y_{T_{1}}=y_{R_{2}}=20 \mathrm{~m}$, and $y_{T_{2}}=y_{R_{1}}=10 \mathrm{~m}$. For the reference model, all theoretical results have been obtained by choosing the following parameters: $\gamma_{T}=90^{\circ}, \gamma_{R}=90^{\circ}, \varphi_{v}^{T}=0^{\circ}$, $\varphi_{v}^{R}=180^{\circ}$, and $f_{T_{\max }}=f_{R_{\max }}=91 \mathrm{~Hz}$. The Rice factor $c_{R}$ was chosen from the set $\{0,0.5,1\}$. The scatterers are uniformly distributed over the considered rectangular areas. The $L_{p^{-}}$ norm method has been applied to optimize the simulation model parameters by using a finite number of scatterers (cisoids). For the simulation model, we use $M \times N=50 \times 25$ scatterers (cisoids) within the rectangle on the left-hand side as well as on the right-hand side.

In Figure 3, the absolute value of the $2 \mathrm{D}$ space CCF $\left|\rho_{11,22}\left(\delta_{T}, \delta_{R}\right)\right|$ of the reference model is presented for the NLOS propagation scenario $\left(c_{R}=0\right)$. The results have been obtained by using (30). From Figure 3 , we can observe that the $2 \mathrm{D}$ space CCF decreases as the antenna element spacings 


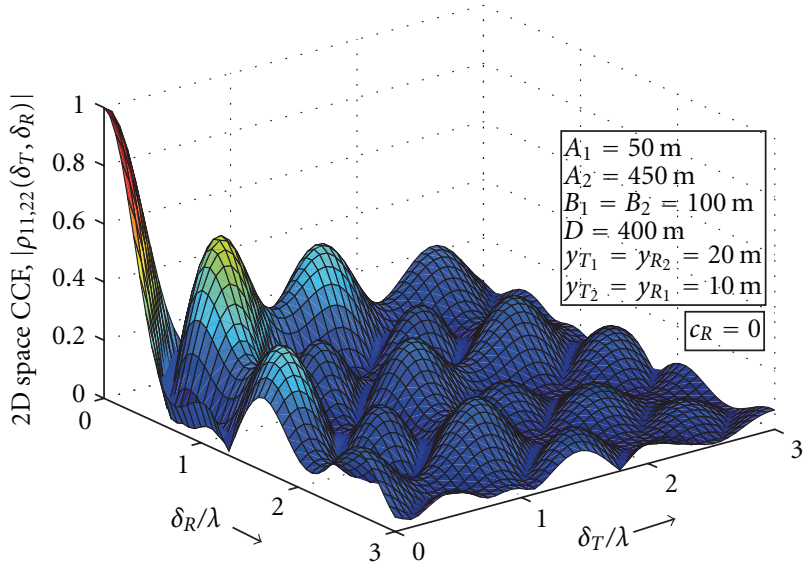

Figure 3: Absolute value of the $2 \mathrm{D}$ space CCF $\left|\rho_{11,22}\left(\delta_{T}, \delta_{R}\right)\right|$ of the reference model for a NLOS propagation scenario $\left(c_{R}=0\right)$.

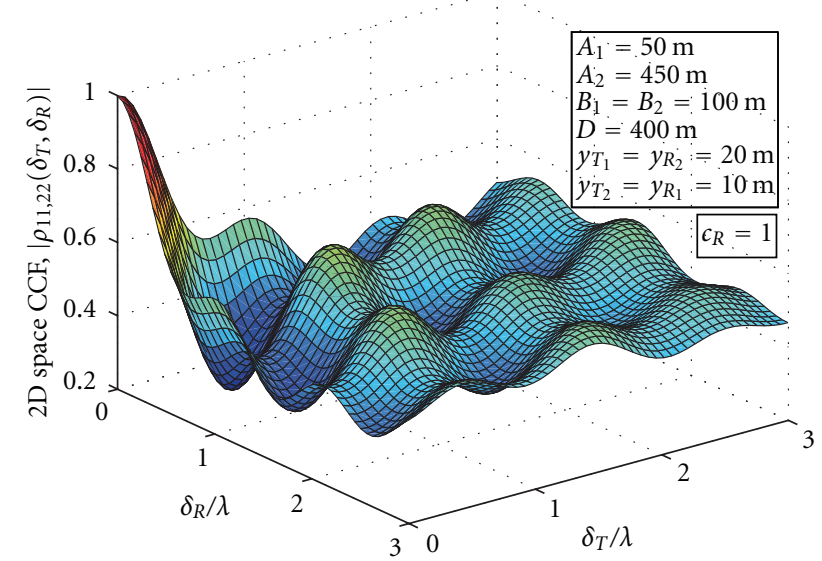

FIgURe 4: Absolute value of the $2 \mathrm{D}$ space CCF $\left|\rho_{11,22}\left(\delta_{T}, \delta_{R}\right)\right|$ of the reference model for a LOS propagation scenario $\left(c_{R}=1\right)$.

increase. For comparison reasons, the absolute value of the $2 \mathrm{D}$ space CCF $\left|\rho_{11,22}\left(\delta_{T}, \delta_{R}\right)\right|$ is depicted in Figure 4 for a LOS propagation scenario $\left(c_{R}=1\right)$. From Figure 4 , one can see that the channel transfer functions $H_{k l}\left(f^{\prime}, t\right)$ and $H_{k^{\prime} l^{\prime}}\left(f^{\prime}, t\right)$ are highly correlated over a large range of antenna element spacings $\delta_{T}$ and $\delta_{R}$. This can be concluded from the fact that even for large antenna element spacings, for example, $\delta_{T}=\delta_{R}=3 \lambda$, the absolute value of the $2 \mathrm{D}$ space CCF $\left|\rho_{11,22}\left(\delta_{T}, \delta_{R}\right)\right|$ equals approximately one half of its maximum value. Comparing Figures 3 and 4 shows that by increasing the Rice factor $c_{R}$, the $2 \mathrm{D}$ space CCF also increases.

Figures 5 and 6 illustrate the TF-CCFs of the reference model under NLOS and LOS propagation conditions, respectively. From Figure 5, we can observe that the TF-CCF decreases as the time and frequency lags increase in NLOS propagation environments. A comparison of Figures 5 and 6 shows that the absolute value of the TF-CCF under LOS conditions is in general higher than under NLOS.

Figure 7 depicts the absolute value of the temporal ACF $\left|r_{k l}(\tau)\right|$ according to (32) if both the transmitter and the receiver are moving towards each other. A good match

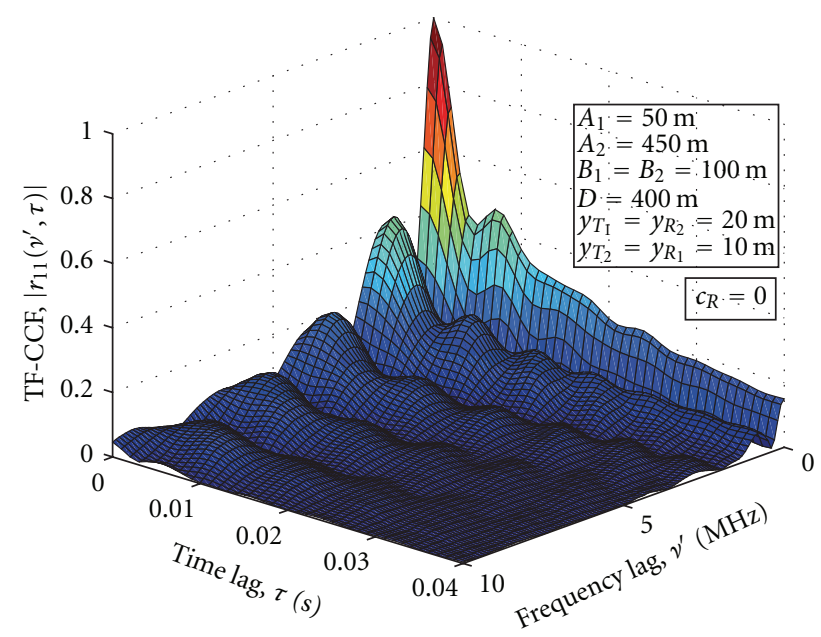

FIGURE 5: Absolute value of the TF-CCF $\left|r_{11}\left(\nu^{\prime}, \tau\right)\right|$ of the reference model for a NLOS propagation scenario $\left(c_{R}=0\right)$.

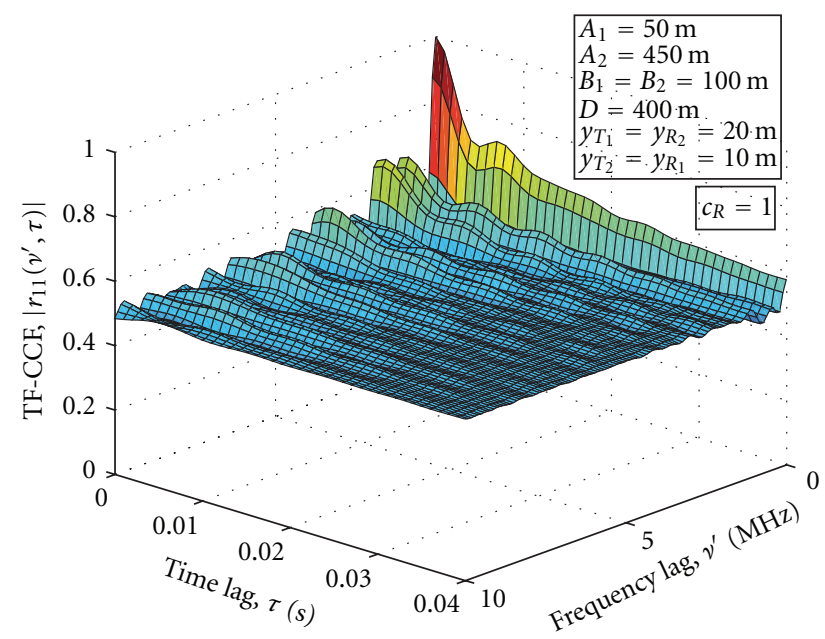

FIgURE 6: Absolute value of the TF-CCF $\left|r_{11}\left(\nu^{\prime}, \tau\right)\right|$ of the reference model for a LOS propagation scenario $\left(c_{R}=1\right)$.

between the temporal ACF of the reference model and that of the simulation model can be observed in Figure 7. This figure demonstrates also that the experimental simulation results of the temporal ACF match very well with the theoretical results.

Finally, Figure 8 illustrates the absolute value of the FCF $\left|r_{k l}\left(v^{\prime}\right)\right|$ for different Rice factors $c_{R}=\{0,0.5,1\}$ if both the transmitter and the receiver are moving towards each other. A close agreement between the reference model and the simulation model can be seen in Figure 8 for all chosen Rice factors. One can realize that the experimental simulation results of the FCF match very well with the theoretical results.

\section{Conclusion}

In this paper, a reference model for a wideband MIMO $\mathrm{C} 2 \mathrm{C}$ channel has been derived by starting from the geometrical street scattering model. Taking both LOS and NLOS 


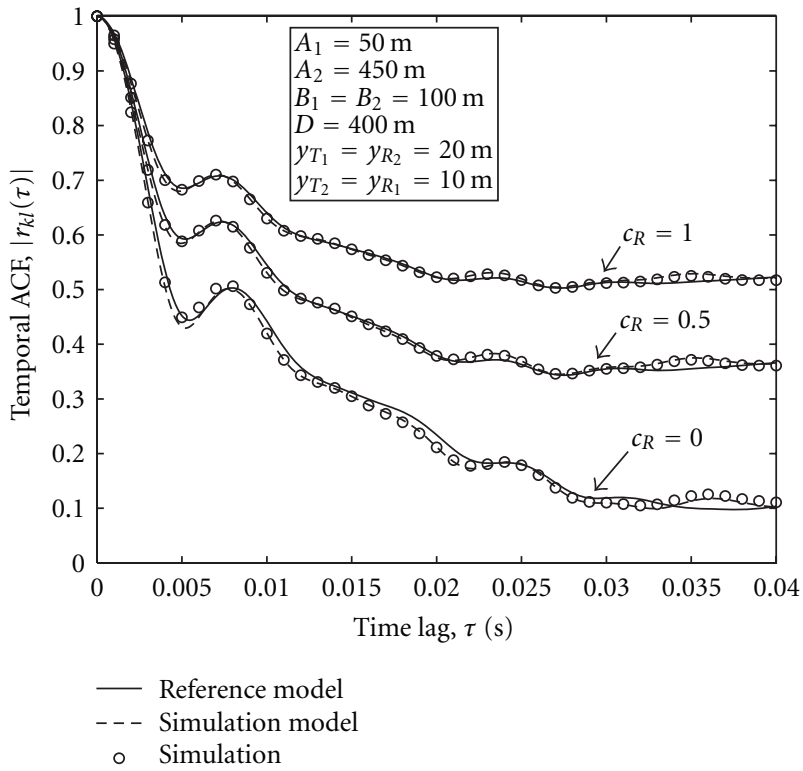

Figure 7: Absolute values of the ACFs $\left|r_{k l}(\tau)\right|$ (reference model) and $\left|\hat{r}_{k l}(\tau)\right|$ (simulation model) for different values of the Rice factor $c_{R} \in\{0,0.5,1\}$.

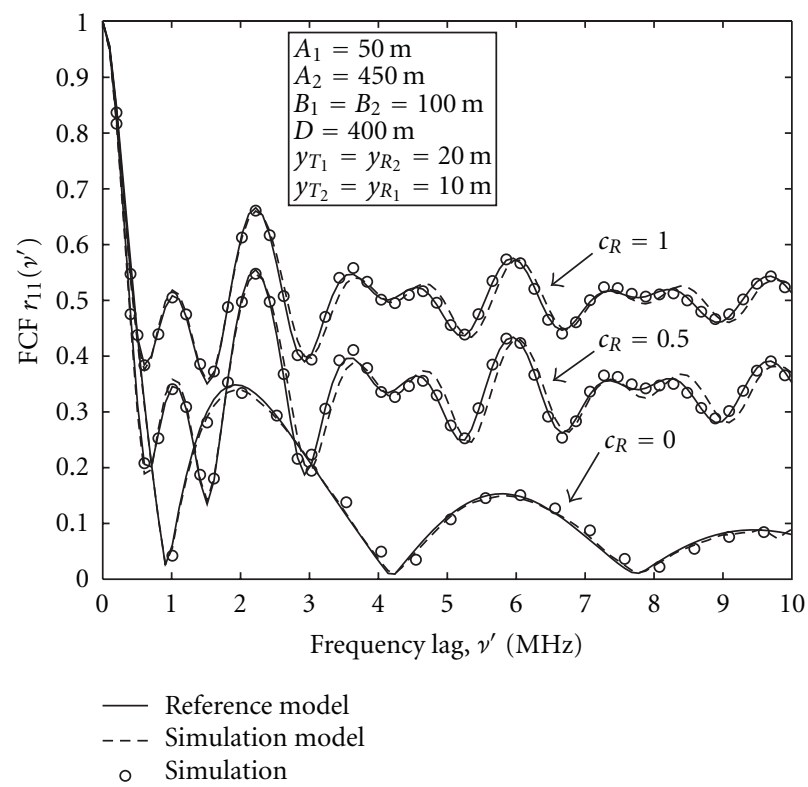

Figure 8: Absolute values of the FCFs $\left|r_{11}\left(\nu^{\prime}\right)\right|$ (reference model) and $\left|\hat{r}_{11}\left(\nu^{\prime}\right)\right|$ (simulation model) for different values of the Rice factor $c_{R} \in\{0,0.5,1\}$.

propagation conditions into account, we have analyzed the $2 \mathrm{D}$ space CCF and the TF-CCF of the reference model. To find a proper simulation model, the SOC principle has been applied. It has been shown that the SOC channel simulator approximates the reference model with high accuracy with respect to the temporal ACF and the FCF. An excellent fitting of the average Doppler shift and the Doppler spread of the reference model to the corresponding quantities of measured channels has validated the usefulness of the proposed reference model. Further extensions of the proposed wideband MIMO C2C channel model incorporating the nonstationarity properties of real-world $\mathrm{C} 2 \mathrm{C}$ channels are planned for future work.

\section{References}

[1] F. Qu, F. Y. Wang, and L. Yang, "Intelligent transportation spaces: vehicles, traffic, communications, and beyond," IEEE Communications Magazine, vol. 48, no. 11, pp. 136-142, 2010.

[2] http://www.ertico.com/.

[3] http://www.car-to-car.org/.

[4] E. Telatar, "Capacity of multi-antenna Gaussian channels," European Transactions on Telecommunications, vol. 10, no. 6, pp. 585-595, 1999.

[5] M. Pätzold, B. O. Hogstad, and N. Youssef, "Modeling, analysis, and simulation of MIMO mobile-to-mobile fading channels," IEEE Transactions on Wireless Communications, vol. 7, no. 2, pp. 510-520, 2008.

[6] M. Pätzold and B. O. Hogstad, "A wideband MIMO channel model derived from the geometric elliptical scattering model," Wireless Communications and Mobile Computing, vol. 8, no. 5, pp. 597-605, 2008.

[7] H. Zhiyi, C. Wei, Z. Wei, M. Pätzold, and A. Chelli, "Modelling of MIMO vehicle-to-vehicle fading channels in T-junction scattering environments," in Proceedings of the 3rd European Conference on Antennas and Propagation (EuCAP '09), pp. 652-656, Berlin, Germany, March 2009.

[8] A. Chelli and M. Pätzold, "A MIMO mobile-to-mobile channel model derived from a geometric street scattering model," in Proceedings of the 4th IEEE International Symposium on Wireless Communication Systems (ISWCS '07), pp. 792-797, Trondheim, Norway, October 2007.

[9] N. Avazov and M. Pätzold, "A geometric street scattering channel model for car-to-car communication systems," in Proceedings of the International Conference on Advanced Technologies for Communications (ATC'11), pp. 224-230, Da Nang City, Vietnam, August 2011.

[10] A. S. Akki and F. Haber, "A statistical model of mobile-tomobile land communication channel," IEEE Transactions on Vehicular Technology, vol. VT-35, no. 1, pp. 2-7, 1986.

[11] A. S. Akki, "Statistical properties of mobile-to-mobile land communication channels," IEEE Transactions on Vehicular Technology, vol. 43, no. 4, pp. 826-831, 1994.

[12] R. Wang and D. Cox, "Channel modeling for ad hoc mobile wireless networks," in Proceedings of the 55th IEEE Vehicular Technology Conference (VTC '02), vol. 1, pp. 21-25, Birmingham, AL, USA, May 2002.

[13] C. S. Patel, G. L. Stüber, and T. G. Pratt, "Simulation of Rayleigh-faded mobile-to-mobile communication channels," IEEE Transactions on Communications, vol. 53, no. 11, pp. 1876-1884, 2005.

[14] A. G. Zajić and G. L. Stüber, "Space-time correlated mobileto-mobile channels: modelling and simulation," IEEE Transactions on Vehicular Technology, vol. 57, no. 2, pp. 715-726, 2008.

[15] X. Cheng, C. X. Wang, D. I. Laurenson, H. H. Ghent, and A. V. Vasilakos, "A generic geometrical-based MIMO mobileto-mobile channel model," in Proceedings of the International Wireless Communications and Mobile Computing Conference (IWCMC '08), pp. 1000-1005, August 2008. 
[16] "IEEE 802.11p, Part 11: wireless LAN medium access control (MAC) and physical layer (PHY) specifications amendment 6: wireless access in vehicular environments, IEEE standards association," June 2010.

[17] "Standard specification for telecommunications and information exchange between roadside and vehicle systems $-5 \mathrm{GHz}$ band dedicated short range communications (DSRC) medium access control (MAC) and physical layer (PHY) specifications," ASTM E2213-03, September 2003.

[18] G. Acosta, K. Tokuda, and M. A. Ingram, "Measured joint Doppler-delay power profiles for vehicle-to-vehicle communications at $2.4 \mathrm{GHz}$," in Proceedings of IEEE Global Telecommunications Conference (GLOBECOM '04), pp. 3813-3817, Dallas, Tex, USA, December 2004.

[19] P. C. F. Eggers, T. W. C. Brown, K. Olesen, and G. F. Pedersen, "Assessment of capacity support and scattering in experimental high speed vehicle-to-vehicle MIMO links," in Proceedings of the IEEE 65th Vehicular Technology Conference (VTC "07), pp. 466-470, April 2007.

[20] I. Sen and D. W. Matolak, "Vehicle-to-vehicle channel models for the 5-GHz band," IEEE Transactions on Intelligent Transportation Systems, vol. 9, no. 2, pp. 235-245, 2008.

[21] Q. Wu, D. W. Matolak, and I. Sen, "5-GHz-band vehicleto-vehicle channels: models for multiple values of channel bandwidth," IEEE Transactions on Vehicular Technology, vol. 59, no. 5, pp. 2620-2625, 2010.

[22] J. Maurer, T. Fügen, and W. Wiesbeck, "Narrow-band measurement and analysis of the inter-vehicle transmission channel at 5.2 GHz," in Proceedings of the 55th Vehicular Technology Conference (VTC'02), vol. 3, pp. 1274-1278, May 2002.

[23] L. Cheng, B. E. Henty, D. D. Stancil, F. Bai, and P. Mudalige, "Mobile vehicle-to-vehicle narrow-band channel measurement and characterization of the $5.9 \mathrm{GHz}$ dedicated short range communication (DSRC) frequency band," IEEE Journal on Selected Areas in Communications, vol. 25, no. 8, pp. 15011516, 2007.

[24] A. Paier, J. Karedal, N. Czink, H. Hofstetter, and C. Dumard, "Car-to-car radio channel measurements at $5 \mathrm{GHz}$ : pathloss, power-delay profile, and delay-Doppler spectrum," in Proceedings of the 4th IEEE International Symposium on Wireless Communication Systems (ISWCS '07), pp. 224-228, Trondheim, Norway, October 2007.

[25] I. Tan, W. Tang, K. Laberteaux, and A. Bahai, "Measurement and analysis of wireless channel impairments in DSRC vehicular communications," in Proceedings of IEEE International Conference on Communications (ICC '08), pp. 4882-4888, May 2008.

[26] J. Kunisch and J. Pamp, "Wideband car-to-car radio channel measurements and model at $5.9 \mathrm{GHz}$," in Proceedings of the 68th IEEE Vehicular Technology (VTC'08), pp. 1-5, September 2008.

[27] O. Renaudin, V. M. Kolmonen, P. Vainikainen, and C. Oestges, "Wideband measurement-based modeling of inter-vehicle channels in the $5 \mathrm{GHz}$ band," in Proceedings of the 5th European Conference on Antennas and Propagation (EUCAP '11), pp. 2881-2885, April 2011.

[28] A. Chelli and M. Pätzold, "A wideband multiple-cluster MIMO mobile-to-mobile channel model based on the geometrical street model," in Proceedings of IEEE 19th International Symposium on Personal, Indoor and Mobile Radio Communications (PIMRC '08), pp. 1-6, Cannes, France, September 2008.
[29] A. G. Zajić, G. L. Stüber, T. G. Pratt, and S. T. Nguyen, "Wideband MIMO mobile-to-mobile channels: geometrybased statistical modeling with experimental verification," IEEE Transactions on Vehicular Technology, vol. 58, no. 2, pp. 517-534, 2009.

[30] X. Cheng, C. X. Wang, D. I. Laurenson, S. Salous, and A. V. Vasilakos, "An adaptive geometry-based stochastic model for non-isotropic MIMO mobile-to-mobile channels," IEEE Transactions on Wireless Communications, vol. 8, no. 9, pp. 4824-4835, 2009.

[31] G. D. Durgin, Space-Time Wireless Channels, Prentice Hall, 2002.

[32] S. H. Kong, "TOA and AOD statistics for down link Gaussian scatterer distribution model," IEEE Transactions on Wireless Communications, vol. 8, no. 5, pp. 2609-2617, 2009.

[33] Y. Ma and M. Pätzold, "A wideband one-ring MIMO channel model under non-isotropic scattering conditions," in Proceedings of IEEE 67th Vehicular Technology Conference (VTC '08), pp. 424-429, Singapore, May 2008.

[34] F. Vatalaro, "Doppler spectrum in mobile-to-mobile communications in the precense of three-dimensional multipath scattering," IEEE Transactions on Vehicular Technology, vol. 46, no. 1, pp. 213-219, 1997.

[35] M. Pätzold, Mobile Radio Channels, John Wiley \& Sons, Chichester, UK, 2nd edition, 2011.

[36] A. Papoulis and S. U. Pillai, Probability, Random Variables and Stochastic Processes, McGraw-Hill, New York, NY, USA, 4th edition, 2002.

[37] M. Pätzold and B. Talha, "On the statistical properties of sum-of-cisoids-based mobile radio channel simulators," in Proceedings of the 10th International Symposium on Wireless Personal Multimedia Communications (WPMC '07), pp. 394400, Jaipur, India, December 2007.

[38] C. A. Gutiérrez and M. Pätzold, "The design of sum-ofcisoids Rayleigh fading channel simulators assuming nonisotropic scattering conditions," IEEE Transactions on Wireless Communications, vol. 9, no. 4, pp. 1308-1314, 2010. 

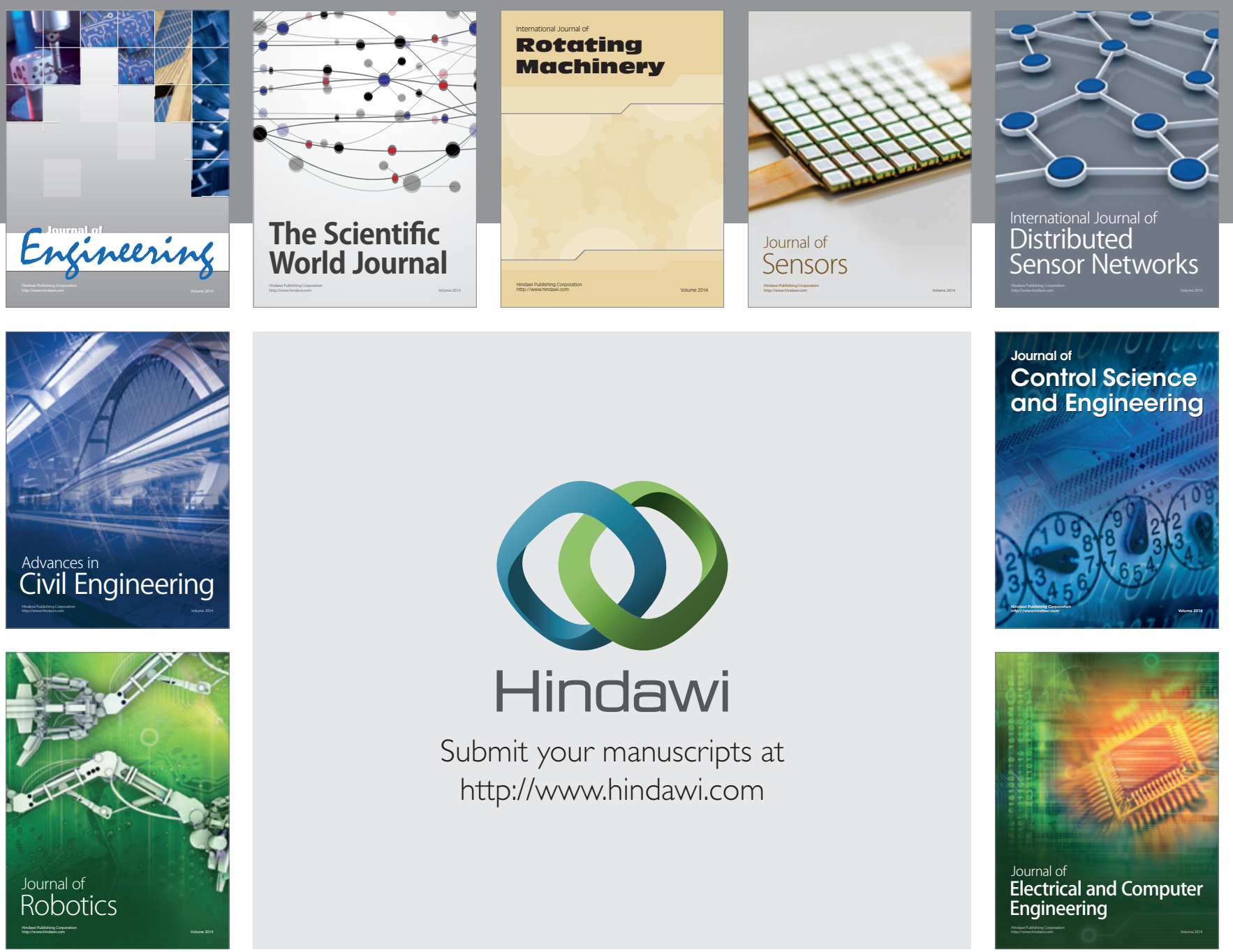

Submit your manuscripts at

http://www.hindawi.com
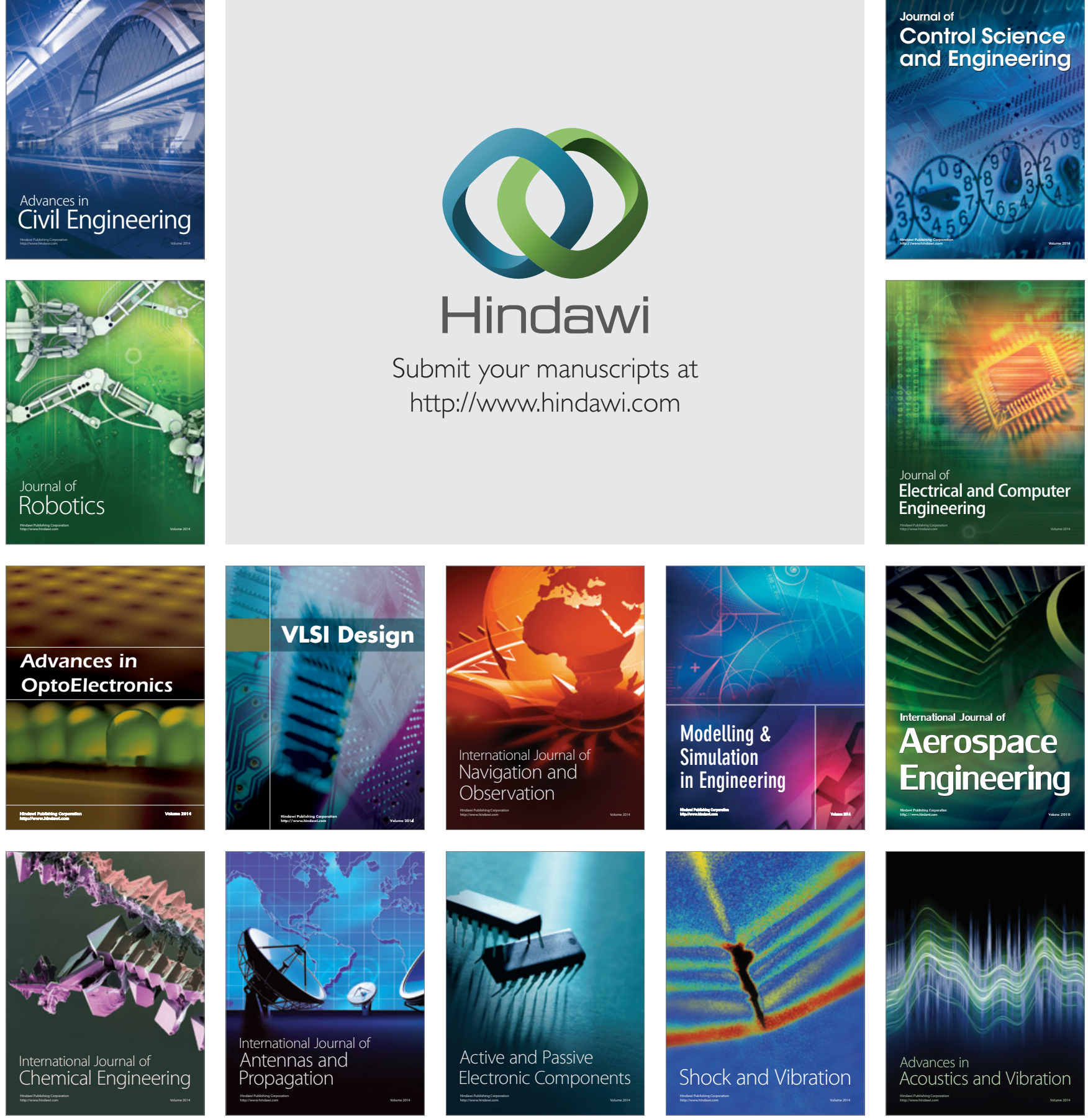